\title{
Designing and Analyzing the Structure of DT-STXB Fusion Protein as an Anti- tumor Agent: An in Silico Approach
}

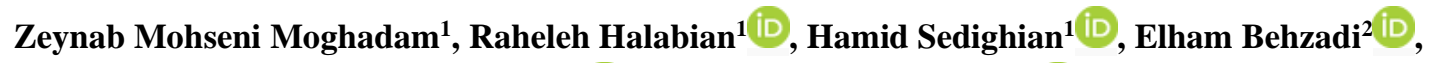 \\ Jafar Amani*1 iD, Abbas Ali Imani Fooladi*1 iD \\ 1. Applied Microbiology Research Center, Systems Biology and Poisonings Institute, Baqiyatallah University of Medical Sciences, Tehran, Iran \\ 2. Department of Microbiology, College of Basic Sciences, Shahr-e-Qods Branch, Islamic Azad University, Tehran, Iran
}

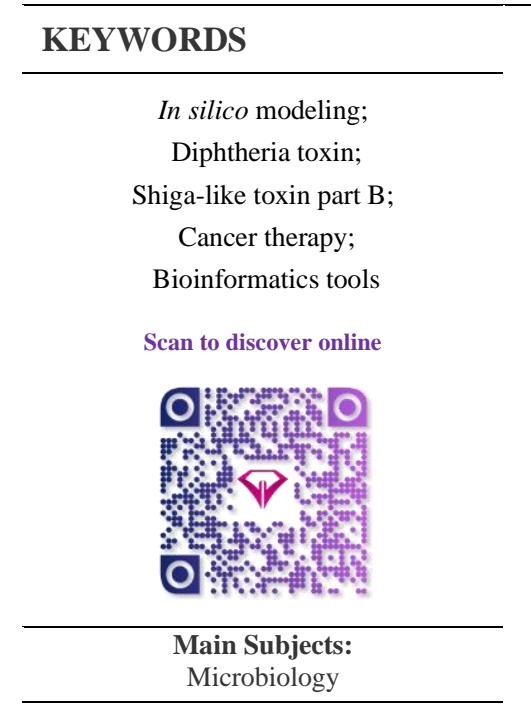

Received 06 Jan 2019;

Accepted 22 Aug 2019;

Published Online 22 Sep 2019;

10.30699/ijp.2019.101200.2004 PMCID:

PMID:

\begin{abstract}
Background \& Objective: A main contest in chemotherapy is to obtain regulator above the biodistribution of cytotoxic drugs. The utmost promising strategy comprises of drugs coupled with a tumor-targeting bearer that results in wide cytotoxic activity and particular delivery. The B-subunit of Shiga toxin (STxB) is nontoxic and possesses low immunogenicity that exactly binds to the globotriaosylceramide (Gb3/CD77). Gb3/CD77 extremely expresses on a number of human tumors such as pancreatic, colon, and breast cancer and acts as a functional receptor for Shiga toxin (STx). Then, this toxin can be applied to target Gb3-positive human tumors. In this study, we evaluated DT390-STXB chimeric protein as a new anti-tumor candidate via genetically fusing the DT390 fragment of DT538 (Native diphtheria toxin) to STxB.
\end{abstract}

Methods: This study intended to investigate the DT390- STxB fusion protein structure in silico. Considering the Escherichia coli codon usage, the genomic construct was designed. The properties and the structure of the protein were determined by an in silico technique. The mRNA structure and the physicochemical characteristics, construction, and the stability of the designed chimeric protein were analyzed using computational and bioinformatics tools and servers. Hence, the GOR4 and I-TASSER online web servers were used to predict the secondary and tertiary structures of the designed protein.

Results: The results demonstrated that codon adaptation index (CAI) of dt390-stxB chimeric gene raised from 0.6 in the wild type to 0.9 in the chimeric optimized gene. The mfold data revealed that the $d t 390-s t x B$ mRNA was completely stable to be translated effectively in the novel host. The normal activity of the fusion protein determined by considering the secondary and tertiary structure of each construct. Energy calculation data indicated that the thermodynamic ensemble for mRNA structure was $-427.40 \mathrm{~kJ} / \mathrm{mol}$. The stability index (SI) of DT390-STxB was 36.95, which is quite appropriate to preserve the stability of the construct. Ultimately, the DT390-STxB was classified as a steady fusion protein according to the Ramachandran plot.

Conclusion: Our results showed that DT390-STXB was a stable chimeric protein and it can be recruited as a candidate of novel anti-tumor agents for the development of breast cancer treatment.

\footnotetext{
Corresponding Information:

Abbas Ali Imani Fooladi, Applied Microbiology Research Center, Systems Biology and Poisonings Institute, Baqiyatallah University of Medical Sciences, Tehran, Iran Email: imanifouladi.a@bmsu.ac.ir

Jafar Amani, Applied Microbiology Research Center, Systems Biology and Poisonings Institute, Baqiyatallah University of Medical Sciences, Tehran, Iran Email: jafar.amani@gmail.com

Copyright ( $\odot$ 2019. This is an open-access article distributed under the terms of the Creative Commons Attribution- 4.0 International License which permits Share, copy and redistribution of the material in any medium or format or adapt, remix, transform, and build upon the material for any purpose, even commercially.
}

\section{Introduction}

Tumors in cancer patients, which have been cured by surgery, chemotherapy, and radiotherapy return in numerous cases and they mostly metastasize $(1,2)$. The general therapy of cancer has been focused on the nonspecific practice of cytotoxic substances for many years to extinguish neoplastic cells. Yet, chemotherapy is confined via parallel impairment to natural tissues, which results in dose-limiting toxicities (3). The confined remedial index of cytotoxic drugs and the capacity of cancer cells to progress resistance to these manufactured medications is essential to expand novel instruments for aggressive malignancies alone or in combination with cytotoxic factors. Anti-tumor action of bacterial toxins has been recognized in numerous studies. Moreover, it has been assumed that the conjugated compounds of bacterial toxins and ligands kill several kinds of tumors (4-8). Thus, in this 
study we selected diphtheria toxin (DT) and the B-subunit of Shiga toxin (STxB) as anti-tumor agents. Native DT is composed of a 538-amino acid protein with three domains; part $\mathrm{A}$ is the enzymatically active domain, part $\mathrm{B}$ has a hydrophobic domain at the $\mathrm{N}$-terminal portion, and the $\mathrm{C}$ terminal portion of fragment $\mathrm{B}$ is the receptor-binding domain. DT intoxicates susceptible eukaryotic cells via receptor-mediated endocytosis (9). As soon as internalized within an acidic vesicle, the enzymatically active fragment A portion is released into the cytosol. Protein synthesis is repressed by part A-catalyzed adenine diphosphate (ADP) ribosylation of elongation factor 2 , and eventually leads to cell death. This is an effective and capable procedure, likewise, the single molecule of DT can impede up to 2000 ribosomes/min in cell-free systems (10).

The Shiga toxins attached to the AB5 toxins, with an enzymatically active A segment and a nontoxic B segment, are responsible for binding to cellular receptors. The B moiety comprises of five similar B subunits $(7.7 \mathrm{kDa})$ establishing a pentameric circle nearby the central pore in which the C-terminus of the A moiety is attached. Each Bsubunit anchorages three determined binding sites that exactly interrelate with the glycosphingolipid (Gb3). So, each $\mathrm{B}$ moiety can possibly interact with up to $15 \mathrm{~Gb} 3$ molecules, leading to high-affinity binding (11). In humans, expression of $\mathrm{Gb} 3$ is limited to apparent cell types. Moreover, a distinct inclination towards $\mathrm{Gb} 3$ overexpression in tumor tissues compared with the normal tissue has been observed in several cancers such as pancreas, breast, and ovary and also in malignant meningioma, glioma, and acute nonlymphocytic leukemia $(6,11-17)$.

In this study, we designed and constructed a DT-STxB chimeric protein and its fusion protein linked by a hydrophobic linker specified as a candidate for cancer therapy.

\section{Materials and Methods \\ Designing the Chimeric Construct}

The amino acid sequences of DT and STxB proteins were retrieved from Uniprot database. The selected sequences for designing chimeric construct were DT (accession No. Q6KE85) and STxB (accession No. Q7BQ98). The DT consists of 193-amino acid N-terminal A-chain, the 342-amino acid B-chain with a hydrophobic translocation enhancing region, a disulfide bond among chains A and B with the deletion of 145-amino acid innate binding region. In order to construct DT390-STxB fusion, DT was fused to the amino acid sequence of STxB (21-89) as a binding fragment for the cellular receptors. Two parts of the fusion protein were linked using a hydrophobic GGGSGGGSGGGS amino acid linker. To optimize the synthetic gene, in silico analysis was performed using online databases such as Gene Bank codon database, the codon database, and Swiss-Prot reverse translation online tool; gene designer software was also recruited. Furthermore, several hydrophobic linkers were examined by the GOR4 tool (18) to separate two functional parts of the chimeric protein. The chimeric gene was designed for cloning and expression in Escherichia coli (E.coli) using the Java codon optimization tool (JCat) (http://www .jcat.de/ ) and optimizer web server $(19,20)$. VaxiJen server (www.ddg-pharmfac.net/vaxijen) (21) was recruited to predict the immunogenicity of the whole antigen. The chimeric gene was synthesized by Biomatik Company (Ontario, Canada).

\section{RNA Secondary Structure Analysis}

The mRNA secondary structure of the chimeric gene was analyzed using the mfold server (http://mfold.rna. albany .edu/ ) (22).

\section{Chimeric protein confidants}

Physiochemical characterization, theoretical isoelectric point ( $\mathrm{pI})$, molecular weight, total number of positive and negative residues, extinction coefficient $(\mathrm{E})$, instability index (II), aliphatic index (AI), and grand average hydropathy (GRAVY) of the chimeric protein were attained using Expasy ProtParam server (web.expasy.org/ protparam/) (23).

\section{Protein Secondary Structure}

Prediction of secondary structure of DT-STxB fusion protein sequence was made using GOR secondary structure prediction method version IV (http://gor.bb.iastate.edu/) (24). Furthermore, the functional characteristics of the designed protein such as secondary structure, the regions lacking normal structure, coiled-coil domains, sections with low-complexity, transmembrane (TM) helices, the solvent accessible surface area (SASA), and the sites with disulfide bridges were also assessed.

\section{D Structure Prediction}

Further analysis of tertiary structural stability of the recombinant DT390-STxB protein was done by the ITASSER online server (http://zhanglab.ccmb.med. umich.edu/I-TASSER) $\quad$ (25-27), Phyre version 0.2 (http://www.sbg.bio.ic.ac.uk/phyre2 ) (28), and online web servers separately, which generates 3D models along with their confidence score (C-Score).

Energy minimization was confirmed by examining the stability of 3D structure of the chimeric protein recruiting Swiss-PdbViewer (aka DeepView) (29). To assess the availability of the amino acids in the protein, the online ASA program was used (30).

\section{Evaluation of Tertiary Structure}

Following generating 3D model, the Swiss PdbViewer that includes a version of the GROMOS96 43B1 force field was performed for energy minimization. The ProSA-web, Z-scores, and Procheck Ramachandran plot (mordred.bioc. cam.ac.uk/ rapper/rampage.php) were performed for structural evaluation and stereo chemical analyses (31). Also, the alignment of the query and template sequences and the assessment of the generated models were carried out recruiting the Swiss-PdbViewer. Furthermore, the solubility of the DT390-STxB fusion protein was evaluated by PROSO online software (https://prosa.services.came .sbg.ac.at/prosa.php 2)(32).

\section{Protein}

Prediction of Antigenic Properties of the Chimeric

VaxiJen web server (www.ddg-pharmfac.net/vaxijen) (21) was applied for predicating antigenic properties of the chimeric protein based on the physicochemical properties. 


\section{Results}

INNO The design and analysis of chimeric protein properties of 390 residues from $\mathrm{N}$-terminal of DT (lacking 21 amino acids of signal sequence from the $\mathrm{N}$-terminal of the protein) was selected from online gene banks and fused to the amino acid sequence of STXB. Several hydrophobic linkers were tested to choose the top linker which sustains functionality of the standard structure of the two parts of the recombinant protein. Finally, GGGGSGGGGSGGGGS sequence was designated as the linker to maintain the flexibility of the construct (Figure 1A).

Afterwards, the amino acid sequence was backtranslated and nucleic acid codons were adjusted based on the codon, considering $E$. coli as the expression host. The chimeric gene exposed a codon adaptation index (CAI) of 0.62 in comparison to the wild type gene that was $50.15 \%$. The GC\% codon usage bias in E. coli was augmented by upgrading GC\% and CAI to $50.15 \%$ (GC\% of E. coli is nearby 50 ) and 0.62 , respectively. The CAI of $>0.8$ was considered statistically significant in terms of high gene expression level (Figure 1B).

Furthermore, the essential restriction enzyme sites (EcoRI and HindIII) were introduced at the ends of the sequence for cloning purposes.

\section{The mRNA Structure Prediction}

The secondary structure of the $d t 390$-stxB mRNA was predicted using the online software mfold. To specify the anticipated folding of the designed gene, a genetic algorithm-based RNA secondary structure prediction was associated with comparative sequence analysis. The folding of $5^{\prime}$ terminal region of the gene had the same structure as the bacterial gene. The minimum free energy (MFE) was estimated for RNA secondary structure; all 29 structural elements recovered in this study had the same the folding as RNA constructs. The obtained data demonstrated that the mRNA had the sufficient stability for the effective translation in the novel host.

Results are demonstrated in Figure 2 and Table 1. The free energy of thermodynamic cooperative units associated with this structure was $\Delta \mathrm{G}=-427.40 \mathrm{~kJ} / \mathrm{mol}$.

Random coils established to be recurrent 189,81 extended strand and 189 alpha helix (Table 2 and Figure 1C).

\section{Chimeric Protein Properties}

ProtParam web server was used to reveal the physiochemical properties of the DT390-STxB sequence. The number of amino acids was found to be 443 , the average molecular weight was $53 \mathrm{kDa}$, and the $\mathrm{pI}$ was 5.11. In addition, the total number of positively charged residues (Arg+Lys) was 42 and total number of negatively charged residues (Asp+Glu) was 56. The instability index of DT390-STxB was computed by the ProtParam web server, which appeared to be 36.95 (Table 3, 4).

Table 1. Thermodynamic details related to 5' end of chimeric construct DT390-STxB mRNA. According to the minimum free energy $(\Delta \mathrm{G})$ of the $5^{\prime}$ end of chimeric mRNA, their initial ATG is constrained in hairpin loop structure.

\begin{tabular}{ccc}
\hline Structural Element & $\boldsymbol{\Delta G ( D T 3 9 0 - S T X B )}$ & Information(DT390-STXB) \\
\hline Helix & -5.50 & 3 base pairs. \\
\hline Hairpin loop & 5.40 & Closing pair is U335-A340 \\
\hline Stack & -2.10 & External closing pair is U2-A274 \\
\hline Stack & -2.20 & External closing pair is G3-C273 \\
\hline Stack & -2.40 & External closing pair is U4-A272 \\
\hline
\end{tabular}

Table 2. Arrangement of secondary structure of origin and chimeric protein

\begin{tabular}{cccc}
\hline Protein & Extended Strand & Alpha Helix & Random Coil \\
\hline DT390-STXB & 81 & 173 & 189 \\
\hline DT390 & 52 & 160 & 147 \\
\hline STxB & 26 & 14 & 29 \\
\hline
\end{tabular}

Table 3. Parameters calculated by ExPSy's ProtParam tool

\begin{tabular}{cccccccccc}
\hline Sequence length & Mw & TpI & $-\mathbf{R}$ & $+\mathbf{R}$ & EC & II & AI & GRAVY \\
\hline 443 & 53645.38 & 5.11 & 56 & 42 & 49850 & 36.95 & 78.13 & -0.312 \\
\hline
\end{tabular}

*Mw, Molecular weight; T pI, Theoretical Isoelectric point; -R, Number of negative charged residues; +R, Number of positive charged residues; EC, Extinction coefficient at $280 \mathrm{~nm}$; II, Instability index; AI, Aliphatic index; GRAVY, Grand Average Hydropathy. 


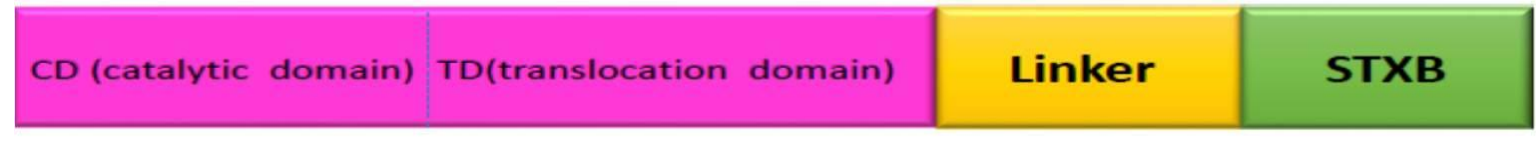

MSSKSFVMENESSYHGTKPGYVDSIQKGIQKPKSGTQGNYDDDWKGEYSTDNKYDAAGYSVDNENPLSG KAGGVVKVTYPGLTKVLALKVDNAET IKKELGLSLTEPLMEQVGTEEFIKRFGDGASRVVLSLPFAEGS SSVEYINNWEQAKALSVELEINEETRGKRGQDAMYEYMAQACAGNRVRRSVGSSLSCINLDWDVIRDKT KTKIESLKEHGPIKNKMSESPNKTVSEEKAKOYLEEFHOTALEHPELSELKTVTGTNPVEAGANYAAWA VNVAQVIDSETADNLEKTTAALSILPGIGSVMGIADGAVHHNTEEIVAQS IALSSLMVAQAIPLVGELV DIGFAAYNEVESI IGGGGSGGGGSGGGGSTPDCVTGKVEYTKYNDDDDTETVKVGDKELETNRWNLQSLL LSAQITGMTVTIKTNACHNGGGESEVIFR

Figure 1A.

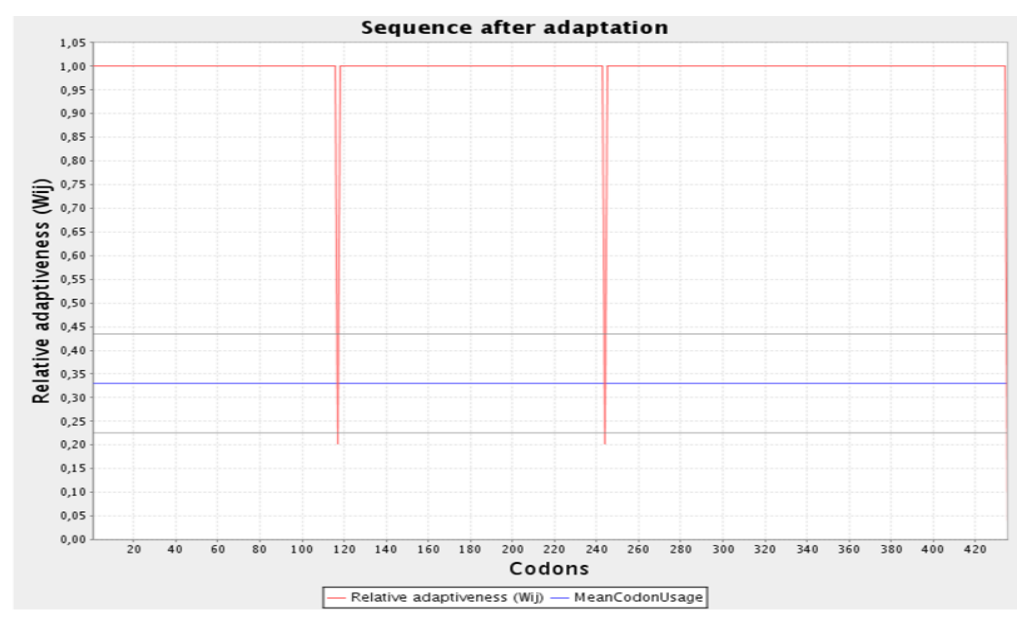

Figure 1B.
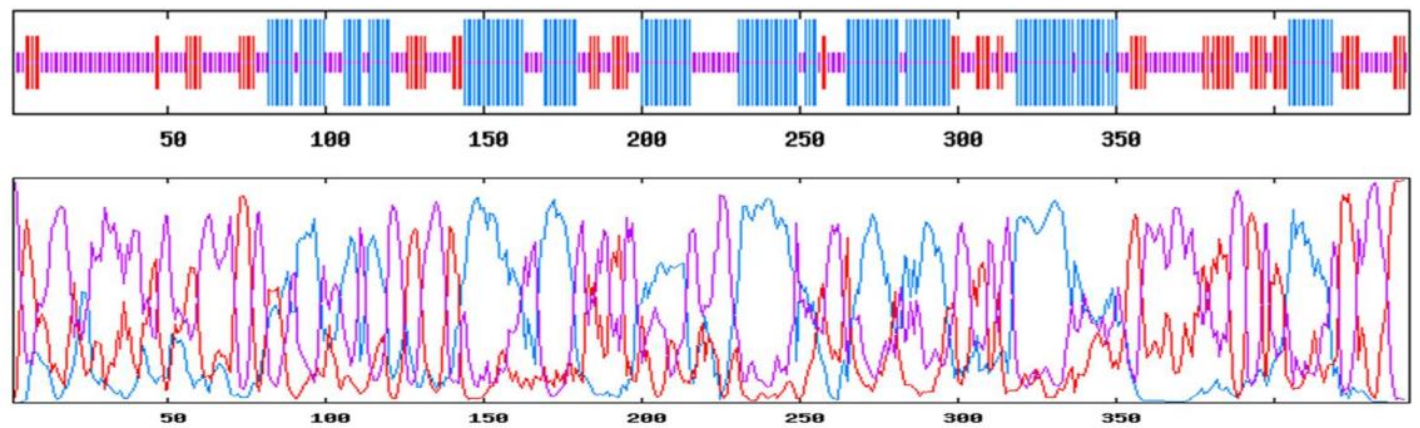

Figure1C.

Fig. 1. A. Sequence alignment and schematic model of hypothetical chimeric protein, which has exposed the construct of DT390 (catalytic and translocate domains) and STxB bound together by the GGGGSGGGGSGGGGS appropriate linker for expression in E. coli. B. Adaptation of codon usage intermittent repartition. The red line demonstrates the codon usage for every codon, which is present in the gene. The blue line shows the mean codon usage in $E$. coli which was assessed for any recognized gene of this organism. The grey lines above and under the blue line display the standard deviation for this mean codon usage in the E. coli. C. Graphical picture of secondary elements in chimeric DT390-STxB protein. The solubility supplies have been categorized by the main polarity and hydrophobic properties of residual patterns. These patterns have exposed that the mean residue accessible surface area (ASA) have given a high solvent convenience value.

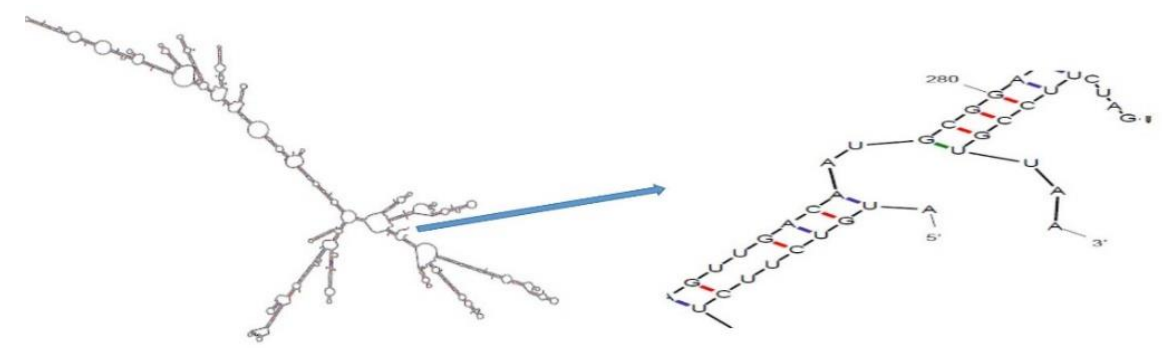

Fig. 2. The mRNA secondary structure of $d t 390-s t x B$ prediction. Predicted structure has a pseudoknot at 5' site of mRNA. $(\Delta \mathrm{G}=-427.40 \mathrm{~kJ} / \mathrm{mol})$ in mfold tool. 
Table4. Amino acid composition of DT390-STxB chimeric protein

\begin{tabular}{cccccc}
\hline Amino acid & No. of residues & Percentage of residues & Amino acid & No. of residues & Percentage of residues \\
\hline Ala (A) & 34 & $7.7 \%$ & Phe (F) & 15 & $3.4 \%$ \\
\hline Arg (R) & 10 & $2.3 \%$ & Pro (P) & 13 & $2.9 \%$ \\
\hline Asn (N) & 24 & $5.4 \%$ & Ser (S) & 39 & $8.8 \%$ \\
\hline Asp (D) & 22 & $5.0 \%$ & $\operatorname{Thr}(\mathrm{T})$ & 30 & $6.8 \%$ \\
\hline Cys (C) & 4 & $0.9 \%$ & $\operatorname{Trp}(\mathrm{W})$ & 5 & $1.1 \%$ \\
\hline Gln (Q) & 14 & $3.2 \%$ & Tyr (Y) & 15 & $3.4 \%$ \\
\hline Glu (E) & 34 & $7.7 \%$ & Val (V) & 35 & $7.9 \%$ \\
\hline Gly (G) & 47 & $10.6 \%$ & Leu (L) & 31 & $7.0 \%$ \\
\hline His(H) & 7 & $1.6 \%$ & Lys (K) & 32 & $7.2 \%$ \\
\hline Ile (I) & 23 & $5.2 \%$ & Met (M) & 9 & $2.0 \%$ \\
\hline
\end{tabular}

However, by considering these details, the chimeric protein is classified as a stable protein. The estimated half-life of this recombinant protein was $30 \mathrm{~h}$ (mammalian reticulocytes, in vitro), >20 h (yeast, in vivo), and $>10 \mathrm{~h}$ (E. coli, in vivo). The grand hydropathicity is considered to be -0.312 .

Secondary and tertiary structures of chimeric protein were predicted to define the final structure of a chimeric protein; the DT390-STxB chimeric protein was submitted to I-TASSER and GOR4 online web tools.
According to C-scores planned through this software, model 1 with a $\mathrm{C}$-score of 0.03 had the maximum confidence among the other models. This was graphically signified in Figure 3.

The profile of energy minimization was considered by Swiss-PdbViewer $-7107.159 \mathrm{kcal} / \mathrm{mol}$, which demonstrated that the recombinant protein DT390STxB had the suitable stability. Also, the structural stability of the protein was evaluated based on the data obtained from the Ramachandran plot (Figure 4).

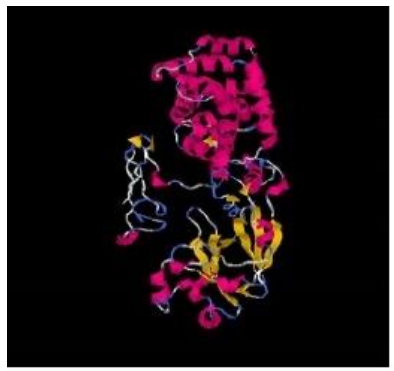

Model 1

C-score $=0.03$

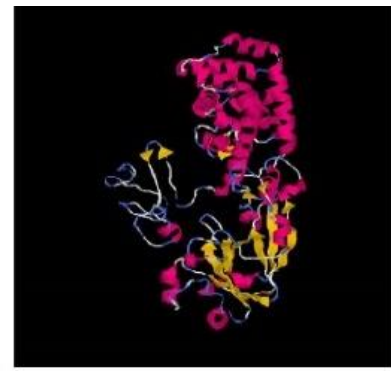

Model 2

C-score $=-0.23$

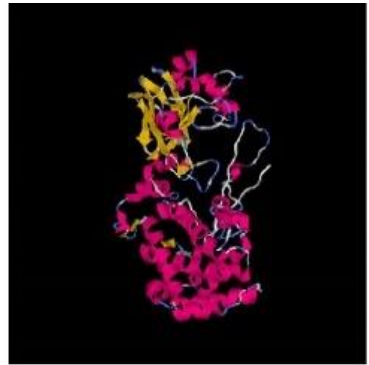

Model 3

C-score $=-0.13$

Figure 3. Tertiary structure prediction. A probabilistic structural model for DT390-STxB chimeric protein by ITASSER server. Based on C-scores, the model 1 has a high confidence score among other models.

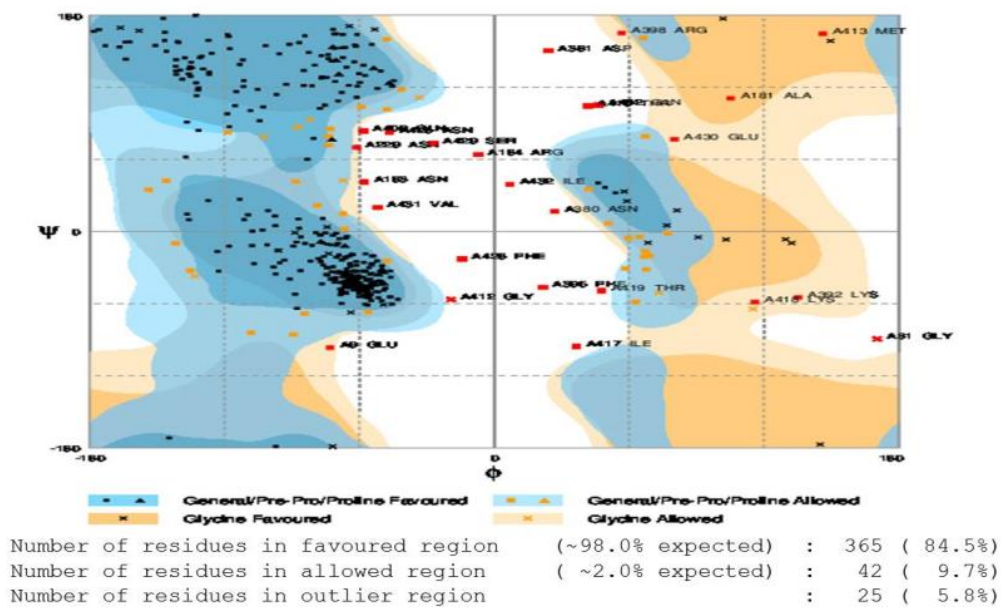

Figure 4. Evaluation of model stability is based on a Ramachandran plot for the DT390-STxB chimeric protein. 


\section{Discussion}

Cancer is the most common cause of death in many developed countries. Today, the targeted therapies like the use of immunotoxins are increased which target specific antigens or receptors on the surface of tumor cells. Immunotoxins are recombinant proteins, which consist of a tumor-specific ligand-like antibody and a toxic protein. Ligand-targeted therapy makes it possible for tumor specificity and limited toxicity and promises for developing novel therapies for cancer treatment. It can carry higher doses of a drug to the tumor tissue and may overcome obstacles presented by cytotoxic chemotherapy (33-40).

Native DT is a 538-amino acid protein consisting of three domains. Part A is the enzymatically active domain, part $\mathrm{B}$ has a hydrophobic domain at the $\mathrm{N}$ terminal portion, and the C-terminal portion of fragment $\mathrm{B}$ is the receptor-binding domain. In this study, we evaluated DT390-STXB chimeric protein as a new anti-tumor candidate via genetically fusing the DT390 fragment of DT538 to STxB (41-43).

In a study carried out by Imani-Fooladi et al., the genetically fused protein called the TGF $\alpha \mathrm{L} 3-\mathrm{SEB}$ fusion protein was designed and evaluated as a novel anti-tumor candidate. This protein was constructed by fusing the third loop of transforming growth factor alpha (TGF $\alpha \mathrm{L} 3$ ) to the staphylococcal enterotoxin type B. The CAI index of the TGF $\alpha$ L3-SEB fusion protein enhanced from 0.5 in the wild type to 0.85 in the chimeric optimized gene. Moreover, the overall guanine-cytosine (GC) content decreased from $45.83 \%$ to $44.06 \%$, hence increasing the overall stability of mRNA of the synthetic gene. The random coil was the second most available structure in this experiment. The pI of the hydrophilic TGF $\alpha$ L3-SEB fusion protein was 7.72. The highest $\mathrm{C}$-score of -0.42 was annotated for tertiary structure of the TGF $\alpha$ L3-SEB protein (44). The comparison between the two chimeric proteins showed that the TGF $\alpha$ L3-SEB and DT390-STxB constructs are stable enough and have sufficient affinity to the overexpressed cancer cell receptors.

In another study by Keshtvarz et al., PE38-P4A8 chimeric immunotoxin was designed and evaluated. The GC content and codon bias of both wild and synthetic form of the protein were assessed and the results for optimized gene showed no rarely used codon, and the codon bias was demonstrated in E. coli as the bacterial host. The optimized GC content was about $54.2 \%$ and the CAI value was 0.94 revealing the high and stable expression in bacterial cells. The highest $\mathrm{C}$-score of -3.36 observed in tertiary structure of the fusion protein. For isolating two parts of the proteins, the ASGGPE and $(\mathrm{G} 4 \mathrm{~S})_{3}$ linkers were recruited (45).

In silico studies are able to complete transcriptional and translational gene fusion, other than the quality expression of the suggested concept in host expression vectors. CAI is the main factor used for gene optimization within a range of $0-1$ and an ideal value of 1.0. Subsequently, our objective was to construct a fusion protein that is expressed in $E$. coli as a host expression vector. So, the codon table of $E$. coli was selected for back-translation of the amino acid sequence and ideal expression of the construct. In our gene, CAI index was improved from 0.6 in the wild type sequences to 0.9 in the chimeric optimized gene. Furthermore, the overall GC content was reduced from $50.03 \%$ to $47.58 \%$, which should increase the overall stability of mRNA from the synthetic gene. Codon optimization contributes to improve the expression of the synthetic construct in the desired host vector.

The mRNA structure optimization was established based on the low $\Delta \mathrm{G}$ value and the MFE values of the start codon. These characteristics can support ribosome binding and translation initiation. For prediction of RNA secondary structure, a genetic algorithm-based approach along with relative sequence analysis was recruited to define the prospective folding of the chimeric gene. The $5^{\prime}$ terminus of the gene was folded in a way which is typical for all bacterial gene structures. The MFE for secondary structures formed by RNA molecules was also predicted. The mRNA secondary structure of the chimeric gene was analyzed using the mfold program with the parameters as follows: linear RNA folding at $5 \%$, window $=20$, and max folds $=50$. All 32 structures achieved in this analysis exposed folding of the RNA construct at $37^{\circ} \mathrm{C}$ with initial $\Delta \mathrm{G}$ ranging from -543.80 to $-481.78 \mathrm{kcal} / \mathrm{mol}$. The best structure $(\Delta \mathrm{G}=-427.40$ $\mathrm{kJ} / \mathrm{mol}$ ) is shown in Figure 4. The data showed that the mRNA was stable enough for proper translation in the new host.

ProtParam was used to determine the physiochemical properties of the protein sequence. The results of the primary structure analysis showed that DT390-STxB fusion protein is hydrophilic in nature due to the large number of polar residues. Also, the primary structure analysis suggests that the average molecular weight of DT390-STXB is considered to be $53.64 \mathrm{kDa}$. The $\mathrm{pI}$ of a protein is defined as the $\mathrm{pH}$ at which the net charge on the surface of the protein is zero. At pI, the proteins are stable and dense. The calculated pI value of DT390-STxB is $5.11(\mathrm{pI}<7)$. Although ExPASy's ProtParam computes the extinction coefficient $(\varepsilon)$ for a range of wavelengths $(276,278,279,280$, and $282 \mathrm{~nm})$, extinction constant (K) of DT390-STxB at $280 \mathrm{~nm}$ is $49850 \mathrm{M}^{-1} \mathrm{~cm}^{-1}$ with respect to the low concentration of Cys, Trp, and Tyr, which indicates that this fusion protein cannot be analyzed using UV spectral methods.

The three-dimensional (3D) structure of the proteins is of a major importance to provide insights into their molecular functions. The 3D model of the recombinant DT390-STxB protein was produced using the I-TASSER online server, which generates 3D models along with their C-score, Z-score, RMSD, and TM-score. This server was generated in three models with C-scores as follows: $-2.82,-3.32$, and 0.03 . Among the three models, model one was selected for further analysis as it assigned the highest $\mathrm{C}$-score 
(0.03). The expected TM-score was $0.72 \pm 0.11$, which approved the validity of the model. A TM-score more than 0.5 probabilities accuracy of topology. Subsequently, by generating 3D model, structural assessment and stereo chemical analyses were accomplished using Procheck Ramachandran plot. Energy minimization was determined by analysis of 3D structural stability of the chimeric protein using Swiss-PdbViewer. The proportion of residues was $84.5 \%$ in the favored region, $9.7 \%$ in allowed region, and $5.8 \%$ in the outlier region. Also, the assessment of model stability of the Ramachandran plot indicated that the most residues of the chimeric model are in a stable zone. The model was analyzed by different DT390STXB fusion proteins as a new anti-tumor candidate. Since it is important to establish the structure-function relation of DT390-STxB fusion protein, some experimental studies have already started investigating the DT390-STxB fusion protein by several tools and software.

In this study, we introduce a novel anti-tumor fussion protein. Our bioinformatics results show that this protein was a stable chimeric protein and it can be used for treatment against breast cancer.

\section{Acknowledgements}

This study has been extracted from a PhD's thesis, which supported by applied Biotechnology applied microbiology Research Center, Baqiyatallah University of medical Sciences, Tehran, Iran.

\section{Conflict of Interest}

The authors declared that there is no conflict of interest regarding the publication of this article.

\section{References}

1. Hutchinson AD, Hosking JR, Kichenadasse G, Mattiske JK, Wilson C. Objective and subjective cognitive impairment following chemotherapy for cancer: a systematic review. Cancer treatment reviews. 2012;38(7):926-34. [DOI:10.1016/j.ctrv.2012.05.002] [PMID]

2. Sui X, Chen R, Wang Z, Huang Z, Kong N, Zhang M, et al. Autophagy and chemotherapy resistance: a promising therapeutic target for cancer treatment. Cell death \& disease. 2013;4(10):e838. [DOI:10.1038/cddis.2013.350] [PMID] [PMCID]

3. Sharkey RM, Goldenberg DM. Targeted therapy of cancer: new prospects for antibodies and immunoconjugates. CA: A Cancer Journal for Clinicians. 2006;56(4):226-43. [DOI:10.3322/canjclin.56.4.226] [PMID]

4. Jain KK. Use of bacteria as anticancer agents. Expert Opinion on Biological Therapy. 2001;1(2):291-300. [DOI:10.1517/14712598.1.2.291] [PMID]

5. Frankel AE. Methods for treating acute myeloid leukemia with diphtheria toxin-interleukin-3 conjugates. Google Patents; 2013.

6. Frankel AE. Methods and compositions based on diphtheria toxin-interleukin-3 conjugates. Google Patents; 2015.

7. Prince HM, Newland KM. Denileukin diftitox for the treatment of cutaneous T-cell lymphoma. Expert Opinion on
Orphan Drugs. 2014;2(6):625-34. [DOI:10.1517/21678707.2014.912580]

8. Zhan C, Li C, Wei X, Lu W, Lu W. Toxins and derivatives in molecular pharmaceutics: drug delivery and targeted therapy. Advanced drug delivery reviews. 2015;90:101-18. [DOI:10.1016/j.addr.2015.04.025] [PMID]

9. Schubert I. Diphtheria Toxin Based Molecules as Therapeutic Approaches. Corynebacterium diphtheriae and Related Toxigenic Species: Springer; 2014. p. 277-90. [DOI:10.1007/978-94-007-7624-1_15]

10. Williams D, Parker K, Bacha P, Bishai W, Borowski M, Genbauffe F, et al. Diphtheria toxin receptor binding domain substitution with interleukin-2: genetic construction and properties of a diphtheria toxin-related interleukin-2 fusion protein. Protein engineering. 1987;1(6):493-8. [DOI:10.1093/protein/1.6.493] [PMID]

11. Chan YS, Ng TB. Shiga toxins: from structure and mechanism to applications. Applied microbiology and biotechnology. 2016;100(4):1597-610 [DOI:10.1007/s00253-015-7236-3] [PMID]

12. LaCasse E, Bray M, Patterson B, Lim W-M, Perampalam S, Radvanyi L, et al. Shiga-like toxin-1 receptor on human breast cancer, lymphoma, and myeloma and absence from CD34+ hematopoietic stem cells: implications for ex vivo tumor purging and autologous stem cell transplantation. Blood. 1999;94(8):2901-10.

13. Gaston MA, Pellino CA, Weiss AA. Failure of manganese to protect from Shiga toxin. PloS one. 2013;8(7):e69823. [DOI:10.1371/journal.pone.0069823] [PMID] [PMCID]

14. Haicheur N, Bismuth E, Bosset S, Adotevi O, Warnier G, Lacabanne V, et al. The B subunit of Shiga toxin fused to a tumor antigen elicits CTL and targets dendritic cells to allow MHC class I-restricted presentation of peptides derived from exogenous antigens. The Journal of Immunology. 2000;165(6):3301-8. [DOI:10.4049/jimmunol.165.6.3301] [PMID]

15. Geyer PE, Maak M, Nitsche U, Perl M, Novotny A, SlottaHuspenina J, et al. Gastric adenocarcinomas express the glycosphingolipid Gb3/CD77: Targeting of gastric cancer cells with Shiga toxin B-subunit. Molecular cancer therapeutics. 2016;15(5):1008-17. [DOI:10.1158/15357163.MCT-15-0633] [PMID]

16. Ishitoya $\mathrm{S}$, Kurazono $\mathrm{H}$, Nishiyama $\mathrm{H}$, Nakamura $\mathrm{E}$, Kamoto $\mathrm{T}$, Habuchi $\mathrm{T}$, et al. Verotoxin induces rapid elimination of human renal tumor xenografts in SCID mice. The Journal of urology. 2004;171(3):1309-13. [DOI:10.1097/01.ju.0000100110.11129.85] [PMID]

17. Batisse C, Dransart E, Sarkouh RA, Brulle L, Bai S-K, Godefroy $S$, et al. A new delivery system for auristatin in StxB-drug conjugate therapy. European journal of medicinal chemistry. 2015;95:483-91. [DOI:10.1016/i.eimech 2015.03.047] [PMID]

18. Doolittle ED. Methods in Enzymology, RF. 1996. p. 540-53.

19. Puigbo P, Guzman E, Romeu A, Garcia-Vallve S. OPTIMIZER: a web server for optimizing the codon usage of DNA sequences. Nucleic Acids Res 2007;35:W126-131 [DOI:10.1093/nar/gkm219] [PMID] [PMCID]

20. Puigbo P, Romeu A, Garcia-Vallve S. HEG-DB: a database of predicted highly expressed genes in prokaryotic complete genomes under translational selection. Nucleic Acids Res 
2008;36:D524-527. [DOI:10.1093/nar/gkm831] [PMID] [PMCID]

21. Irini A. D, and Darren R. F. Bioinformatic Approach for Identifying Parasite and Fungal Candidate Subunit Vaccines. The Open Vaccine Journal. 2008; 1: 22-26. [DOI:10.2174/1875035400801010022]

22. Zuker M. Mfold web server for nucleic acid folding and hybridization prediction. Nucleic Acids Res 2003;31:34063415. [DOI:10.1093/nar/gkg595] [PMID] [PMCID]

23. Amala S. In silico Analysis and 3D Modeling of ASAH1 Protein in Farber Lipogranulomatosis. Advanced Biotech 2010;10(6):6-8.

24. Garnier J, Gibrat JF, Robson B. Methods in Enzymology. Ed RFD, editor1996.

25. Zhang Y. I-TASSER server for protein 3D structure prediction [Research Support, Non-U.S. Gov't]. 2008 [cited 9]. 2008/01/25:[40]. Available from: http://www.ncbi. nlm.nih.gov/pubmed/18215316. [DOI:10.1186/1471-21059-40] [PMID] [PMCID]

26. Roy A, Kucukural A, Zhang Y. I-TASSER: a unified platform for automated protein sztructure and function $\begin{array}{lll}\text { prediction. Nat Protoc 2010;5:725-738. } & \text {. }\end{array}$ [DOI:10.1038/nprot.2010.5] [PMID] [PMCID]

27. Roy A, Yang J, Zhang Y. COFACTOR: an accurate comparative algorithm for structure-based protein function annotation. Nucleic Acids Res 2012;40:W471-477. [DOI:10.1093/nar/gks372] [PMID] [PMCID]

28. Kelley LA, Sternberg MJ. Protein structure prediction on the Web: a case study using the Phyre server. Nat Protoc 2009;4:363-371. [DOI:10.1038/nprot.2009.2] [PMID]

29. Guex N, Peitsch MC. SWISS-MODEL and the Swiss-Pdb Viewer: an environment for comparative protein modeling. electrophoresis. [DOI:10.1002/elps.1150181505] [PMID] 1997;18(15):2714-23.

30. Ahmad S, Gromiha M, Fawareh H, Sarai A. ASAView: database and tool for solvent accessibility representation in proteins. BMC bioinformatics. 2004;5(1):51. [DOI:10.1186/1471-2105-5-51] [PMID] [PMCID]

31. Lovell SC, Davis IW, Arendall WB 3rd, de Bakker PI, Word JM, Prisant MG, Richardson JS, and et al. Structure validation by Calpha geometry: phi,psi and Cbeta deviation. Proteins 2003;50:437-450. [DOI:10.1002/prot.10286] [PMID]

32. Wiederstein M, Sippl MJ. ProSA-web: interactive web service for the recognition of errors in three-dimensional structures of proteins. Nucleic Acids Res. 2007;35: 407-10. [DOI:10.1093/nar/gkm290] [PMID] [PMCID]

33. Shaw J, Akiyoshi DE, Arrigo DA, Rhoad AE, Sullivan B, Thomas J, et al. Cytotoxic properties of DAB486EGF and DAB389EGF, epidermal growth factor (EGF) receptortargeted fusion toxins. Journal of Biological Chemistry. 1991;266(31):21118-24

34. Cawley DB, Herschman HR, Gilliland DG, Collier RJ. Epidermal growth factor-toxin A chain conjugates: EGF- ricin $\mathrm{A}$ is a potent toxin while EGF-diphtheria fragment $\mathrm{A}$ is nontoxic. Cell. 1980;22(2):563-70. [DOI:10.1016/00928674(80)90366-9]

35. Takahashi T, Umata T, Mekada E. Extension of juxtamembrane domain of diphtheria toxin receptor arrests translocation of diphtheria toxin fragment A into cytosol. Biochemical and biophysical research communications. 2001;281(3):690-6. [DOI:10.1006/bbrc.2001.4427] [PMID]

36. Akin S, Babacan T, Sarici F, Altundag K. A novel targeted therapy in breast cancer: cyclin dependent kinase inhibitors. J BUON. 2014;19(1):42-6.

37. Alewine C, Hassan R, Pastan I. Advances in anticancer immunotoxin therapy. The oncologist. 2015;20(2):176-85. [DOI:10.1634/theoncologist.2014-0358] [PMID] [PMCID]

38. Allahyari H, Heidari S, Ghamgosha M, Saffarian P, Amani J. Immunotoxin: A new tool for cancer therapy. Tumor Biology. 2017;39(2):1010428317692226. [DOI:10.1177/1010428317692226] [PMID]

39. Chandramohan V, Sampson JH, Pastan I, Bigner DD. Toxin-based targeted therapy for malignant brain tumors. Clinical and Developmental Immunology. 2012;2012. [DOI:10.1155/2012/480429] [PMID] [PMCID]

40. Tinoco G, Warsch S, Glück S, Avancha K, Montero AJ. Treating breast cancer in the 21st century: emerging biological therapies. Journal of Cancer. 2013;4(2):117. [DOI:10.7150/jca.4925] [PMID] [PMCID]

41. Maak M, Nitsche U, Keller L, Wolf P, Sarr M, Thiebaud M, et al. Tumor-specific targeting of pancreatic cancer with Shiga toxin B-subunit. Molecular cancer therapeutics.2011;10(10): 1918-28. [DOI:10.1158/15357163.MCT-11-0006] [PMID]

42. Johansson D, Kosovac E, Moharer J, Ljuslinder I, Brännström T, Johansson A, et al. Expression of verotoxin1 receptor $\mathrm{Gb} 3$ in breast cancer tissue and verotoxin-1 signal transduction to apoptosis. BMC cancer. 2009;9(1):67. [DOI:10.1186/1471-2407-9-67] [PMID] [PMCID]

43. Geyer PE, Maak M, Nitsche U, Perl M, Novotny A, SlottaHuspenina J, et al. Gastric adenocarcinomas express the glycosphingolipid Gb3/CD77: Targeting of gastric cancer cells with Shiga toxin B-subunit. Molecular cancer therapeutics. 2016;15(5):1008-17. [DOI:10.1158/15357163.MCT-15-0633] [PMID]

44. Imani-Fooladi AA, Yousefi F, Mousavi SF, Amani J. In silico design and analysis of TGFal3-seb fusion protein as "a new antitumor agent" candidate by ligand-targeted superantigens technique. Iranian journal of cancer prevention. 2014;7(3):152.

45. Keshtvarz M, Salimian J, Yaseri M, Bathaie SZ, Rezaie E, Aliramezani A, et al. Bioinformatic prediction and experimental validation of a PE38-based recombinant immunotoxin targeting the Fn14 receptor in cancer cells. Immunotherapy. 2017;9(5):387-400. [DOI:10.2217/imt2017-0008] [PMID] 